\title{
Value of screening for deafness in the first year of life
}

\author{
R. BOOTHMAN AND N. ORR \\ From the Social Paediatric and Obstetric Research Unit, Glasgow
}

SUMMARY Despite efforts to screen all babies for deafness within their first year, the handicap is still being detected later than is desirable. This study was designed to evaluate the effectiveness of current audiometric screening programmes. Hearing tests were carried out on 535 infants between the ages of 6 months and one year using conventional tests, which depend on the infants' response to familiar sounds, and pure-tone audiometry. The results reveal inadequacies in both sets of tests which, coupled with a high default rate, may well account for the very small contribution that routine audiometric screening is making to the early diagnosis of deafness.

The importance of early detection of deafness has been stressed by a number of workers (Ewing and Ewing, 1944; Whetnall, 1956; Markides, 1971; Taylor, 1974). These authors point out that if treatment is not initiated before the age of 7 years it may be almost impossible to use residual hearing in subsequent training and education. Indeed, Whetnall goes as far as to suggest that the deaf child should have a hearing aid by the time he starts to crawl. Recognition of the importance of the early detection of deafness has led to the recommendation that all infants should be screened for deafness (Ellis and Mitchell, 1973; Forfar and Arneil, 1973). Efforts to implement this recommendation have been made in the Glasgow area. However, when the source of referral of children requiring special educational help on account of deafness was investigated, it was found that only a few deaf children had come to light as a result of routine screening. This may be a reflection of poor use of the service-a possibility that cannot be discounted in view of the high default rates at routine developmental screening clinics. However, an alternative explanation is that the tests may not have been suitable for screening. The high default rate could be made lower by introducing domiciliary screening, but the validity of test procedures is first worth considering. Tests currently being used for audiometric screening depend on the infants' response to familiar sounds and these are difficult to standardise. The principal items used are a high- and a low-frequency rattle, but these do not

Received 13 December 1977 produce pure tones and indeed cover a wide range of frequencies and have variable outputs which cannot be satisfactorily calibrated (Fig. 1). Furthermore, there may be variations in the distance between the source of sound and the patient, and also the way in which the rattle is shaken. Such possible sources of observer error must cast doubt on the value of this technique in a screening test.

Pure-tone audiometry overcomes many of these variables because both frequency and amplitude can be calibrated. Responses to such pure tones have in the past not been found to be sufficiently satisfactory for the technique to displace the more conventional tests based on sounds familiar to the child (Ewing and Ewing, 1944; Robson, 1970). More acceptable response rates were obtained, however, when toys and puppets were used to camouflage the sound source (Murphy, 1964; Supple and Slater, 1968).

A study comparing conventional tests with modified pure-tone audiometry was therefore carried out on 535 children to evaluate the relative merits of both techniques as screening tests.

\section{Materials and methods}

The parents of all infants (1070) whose ages ranged from 6 months to one year and who lived within the areas served by two child welfare clinics were invited to bring them for a hearing test. Each clinic was chosen because it had room available with a low level of background noise. One of the clinics (A) was sited in an area where services were well utilised, while in the other clinic (B) default rates were 


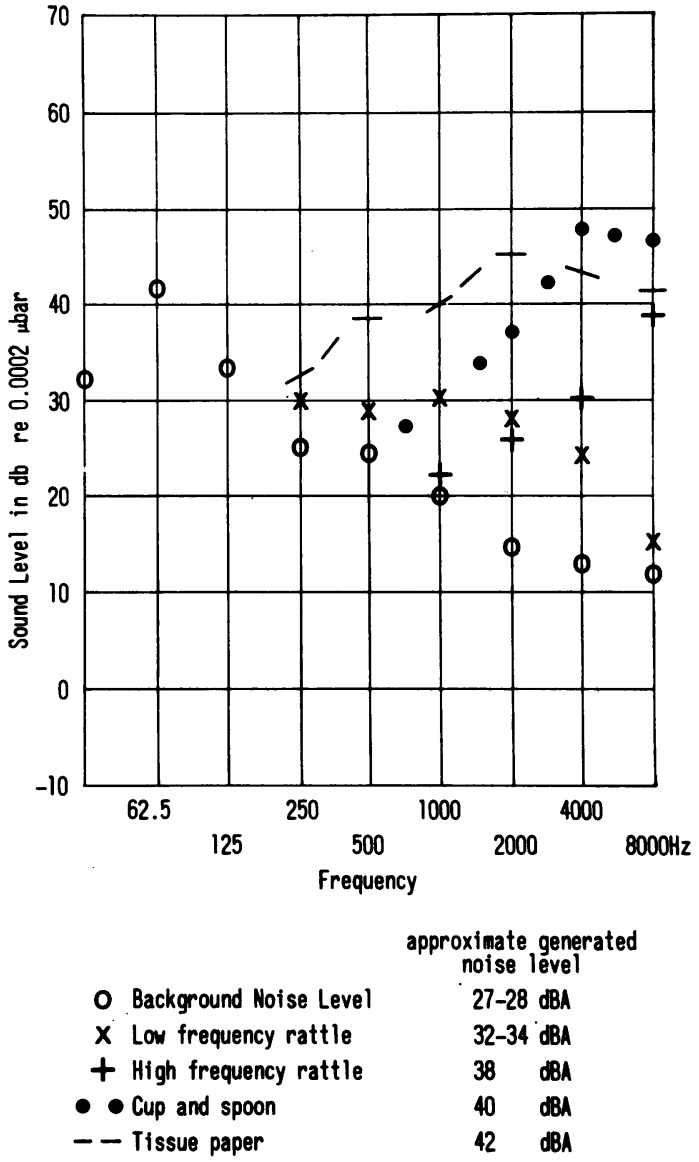

Fig. 1 The wide range of frequencies covered by sounds in the conventional tests.

generally high. Clinic (A) had a much bigger infant population in its catchment area than clinic (B), hence 725 children from clinic (A) and only 345 from clinic (B) were invited to attend.

\section{Technique}

Pure-tone audiometry. The audiometer (Fig. 2) consisted of a pure-tone generator with variable frequency and volume connected by leads to two loudspeakers with a noiseless control switch. The output of the loudspeakers was adjusted to give a nominal $40 \mathrm{~dB}$ or $60 \mathrm{~dB}$ (re. 0.0002 bars to $0.5 \mathrm{~cm}$ and at $200 \mathrm{~Hz}, 1 \mathrm{kHz}$, and $4 \mathrm{kHz}$ ).

The baby was supported on the mother's knee with the loudspeakers at a distance of $0.5 \mathrm{~m}$ on each side (Fig. 3). The tester sat facing the baby and was able to operate the audiometer unobtrusively. Any response to the noise could be clearly seen and interpreted by the observer placed in front of the child. When the child responded to the sounds a toy was offered as a reward.

Conventional tests. As in the case of pure-tone audiometry the child was supported on the mother's knee. Since only a definite turn of the head could be interpreted from behind the child, a second observer interpreted less well-defined responses. The sounds used were high and low frequency rattles, movement of a teaspoon in a cup, rustling of tissue paper, and soft vocal sounds. Fig. 2 shows the wide range of frequencies produced by these test items.

Responses. The responses obtained to both sets of tests were categorised as being either 'definite' or 'ill defined'. Only those infants who turned towards the sound source were regarded as exhibiting a definite response. Any other less definite response was categorised as an 'ill defined' response. The type of response included in this latter category is shown in the Table.

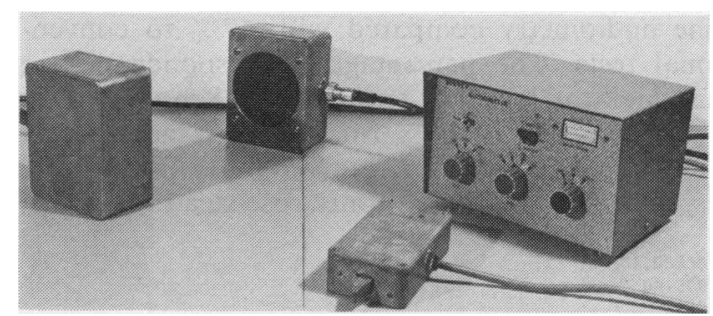

Fig. 2 Portable audiometer with its speakers and noiseless switch.

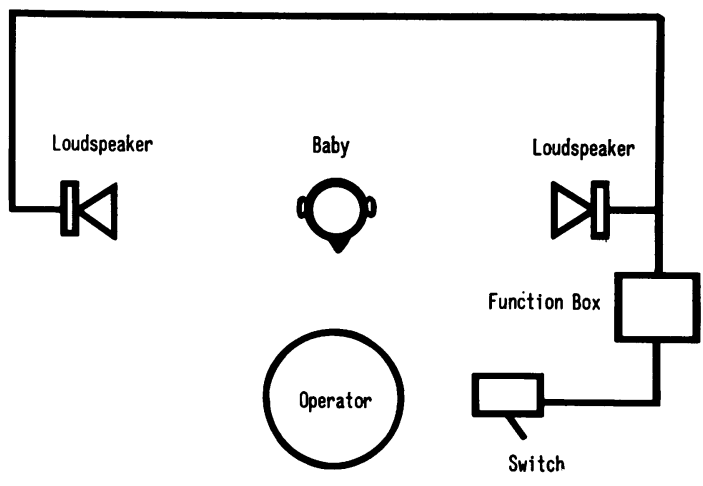

Fig. 3 Diagrammatic representation of location of equipment, patient, and tester using pure-tone audiometry.

\section{Table Summary of 'ill defined' responses}

\footnotetext{
1. Turning of eyes but not head

2. Cessation of activity

3. Repeatedly turning to mother when sound was made 4. Laughing in response to test sounds
} 
Previous studies have accepted only the 'definite' response. We hoped to examine the effect of including all responses to the sounds. Accordingly response rates for both techniques were analysed. Initially only 'definite' responses were accepted, later both 'ill defined' and 'definite' responses were interpreted as indicating the presence of normal hearing.

\section{Results}

Only 535 out of 1070 infants attended the clinic. The results reported here are therefore based on this sample. At all age levels a significantly greater proportion of the children responded satisfactorily to conventional tests than to pure-tone audiometry, irrespective of the criteria used to define such responses. However, the difference was more pronounced when only 'definite' responses were accepted (Fig. 4).

Variations in 'definite' response rates with age. At 6 months of age $35 \%$ of children responded to puretone audiometry compared with $57 \%$ to conventional tests. The percentage of 'responders' rose marginally at 7 months but steeply at 8 months, with a response rate of $54 \%$ for pure-tone audiometry and $77 \%$ for conventional tests. Above this age the response rate using pure tones rose steadily to $73 \%$

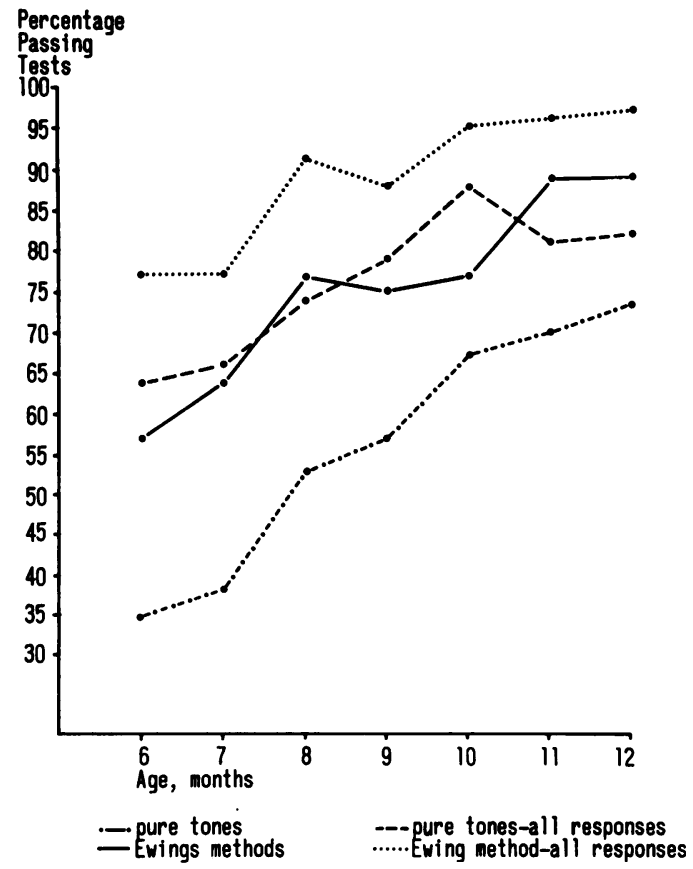

Fig. 4 Percentage of those responding to sounds at various ages. at 12 months of age while the response rate to conventional sounds remained unchanged between the ages of 8 to 10 months and then rose steeply to reach $89 \%$ at both 11 and 12 months.

Variations in total response rates with age. When both 'definite' and 'ill defined' responses were accepted there was a much higher response rate at all age levels irrespective of the techniques used. At 6 months the response rates increased to $64 \%$ for pure-tone audiometry and to $77 \%$ for conventional tests. Once again there was only a marginal increase in response rates at 7 months followed by a steep increase at 8 months to $74 \%$ for pure tones and to $91 \%$ for conventional sounds. After a slight dip at 9 months the response rate using conventional methods rose to a maximum $97 \%$ at 12 months. The response rate using conventional methods rose to a maximum $97 \%$ at 12 months. The response rate to pure-tone audiometry rose to $88 \%$ at 10 months, but fell to $82 \%$ at the 11 and 12 month levels, mainly because it was difficult to hold the attention of children at that age.

Outcome of screening. At the outset it had been decided that all children who failed to respond to conventional tests should be referred for formal testing. However in view of the high failure rate up to the age of 8 months, any child who failed was returned at 9 months and referred only if the results were again unsatisfactory. Any child who failed the tests after this age was referred immediately unless the failure was obviously caused by inattention.

Using this criterion 12 children were referred for full audiometric assessment, 9 of whom were found to have normal hearing, 2 were followed up for one year because they responded poorly to the test stimuli even when the tests were performed under ideal conditions, and one child was referred to the ear nose and throat hospital. Unfortunately, owing to the complexity of the referral system between the primary screening and the child being seen at the ear nose and throat hospital, no information so far is available about the long-term follow-up of this case.

\section{Discussion}

Ewing and Ewing (1944) suggested that only 'definite' responses to test sounds ought to be accepted as indicating the presence of normal hearing. If these criteria are to be adhered to, the findings of this study suggest that neither pure-tone audiometry nor more conventional tests (based on responses to familiar sounds) are suitable screening tests. Hearing tests are generally carried out when 
the child is about 9 months old and at this age such acceptable 'definite' responses were obtained only in $75 \%$ of children for conventional tests and in $57 \%$ for pure-tone audiometry. These figures fall very far short of the figure of $90 \%$ suggested by Holt as the percentage of the normal population who should pass any test designed to be used for screening (Holt, 1974). This of course depends on the assumption that most of those who failed the audiometric screening tests were in fact normal, an assumption which seems justified since not more than one child in the sample was in fact found to be deaf. When the tests were carried out at age 11 or 12 months the response rate became more acceptable but the time taken to overcome inattention made audiometric screening impracticable. If both 'definite' and 'ill defined' responses are accepted the response rate in 10 -month-old children rises to $95 \%$ using conventional tests and to $87 \%$ using pure-tone audiometry tests. These observations raise the question, Can 'ill defined' responses be accepted in the interpretation of routine audiometric screening tests? From the findings of a study carried out by Langford and Bench (1973) it appears that these 'ill defined' responses can be interpreted correctly by relatively inexperienced staff. The technique used in that study was similar to pure-tone audiometry in that the frequency and amplitude of the sounds being produced were standardised and the observer was free to concentrate on the type of response being elicited. It therefore seems reasonable to suggest that all responses to pure-tone audiometry used in this study should be accepted. Conventional hearing tests on the other hand are virtually impossible to standardise on account of the shortcomings of the technique. Routine audiometric screening tests are generally carried out by health visitors who have been instructed in their proper use. The results of this study suggest that the interpretation of either pure-tone audiometry or conventional tests ought to be within the competence of these same workers, but perhaps only 'definite' responses should be accepted.

However before either procedure could be adopted the problem of delivery and acceptance of service has to be considered in view of a default rate of $49 \%$ in this study. Obviously the characteristics of these two clinics are not necessarily representative of other areas in Glasgow, but it is well recognised that attendance rates at child welfare clinics decrease rapidly during the first year of the child's life (Barber et al., 1976). It would be surprising if audiometric screening programmes were not subject to similar default rates. Thus any routine screening programmes would have to be offered on a domiciliary basis and it is unlikely that conditions in many homes would be suitable for audiometric testing. Finally it is doubtful if the resources required to achieve universal screening would be justified for a condition with a probable incidence of 1 per 1000 of the population (Neligan et al., 1974) using techniques which are of such dubious value.

It is possible that a health education programme designed to make parents more aware of the early signs of deafness might prove effective in bringing cases of deafness to light at an early age. However efforts to achieve universal screening in the detection of deafness must not overshadow the need for further research in the field of audiometric techniques in infancy.

We thank all who participated in this study especially the staff of the clinics in which the study was carried out, the children and their parents for their co-operation; Mrs E. McLaughlin for help in assessing children requiring further training; the staff of the Department of Medical Illustration at the Royal Hospital for Sick Children; Dr J. P. Stanfield for advice; and Mrs E. Barry and Mrs A. Sprang for secretarial help.

\section{References}

Barber, J. H., Boothman, R., and Stanfield, J. P. (1976). A new visual chart for pre-school developmental screening. Health Bulletin, 5, 79-91.

Ellis, R. W. B., and Mitchell, R. G. (1973). Disease in Infancy and Childhood, seventh edition. Edited by R. G. Mitchell. Churchill Livingstone: Edinburgh.

Ewing, I. R., and Ewing, A. W. G. (1944). The ascertainment of deafness in infancy and childhood. Journal of Laryngology and Otology, 59, 309-333.

Forfar, J. O., and Arneil, G. C.(1973). Textbook of Paediatrics, p. 1640. Edited by G. Arneil and J. O. Forfar. Churchill Livingstone: Edinburgh.

Holt, K. S. (1974). Infancy and childhood. Lancet, 2, 1057-1060.

Langford, C., and Bench, J. (1973). Neonatal auditory responses assessed by trained and untrained observers: a preliminary study. British Journal of Audiology, 7, 29-37.

Markides, M. (1971). Pre-school audiological services: a suggested outline of early diagnosis of deafness and parent guidance in home training. Sound, 5, 65-69.

Murphy, K. P. (1964). In Research in Deafness in Children. Edited by L. Fisch. Blackwell: Oxford.

Neligan, G., Prudham, D., and Steiner, H. (1974). The Formative Years. Oxford University Press: London.

Robson, J. (1970). Screening techniques in babies. Sound, 4, 91-94.

Supple, M. de M., and Slater, F. (1968). Two new instruments -a doll and a farm-for hearing in young children. Sound, 2, 44-45.

Taylor, I. G. (1974). Deaf children. British Journal of Hospital Medicine, 10, 440-451.

Whetnall, E. (1956). The development of usable (residual) hearing in the deaf child. Journal of Laryngology, and Otology, 70, 630-647.

Correspondence to $\mathrm{Dr}$ R. Boothman, Social Paediatric and Obstetric Research Unit, 64 Oakfield Avenue, Glasgow G12 8LS. 